\title{
Análise da utilização da Grounded Theory (Teoria Fundamentada nos Dados) na produção científica brasileira entre 2008-2012
}

Grounded Theory: analysis of Brazilian scientific production between 2008-2012

\section{Análisis de la aplicación de la Grounded Theory (Teoría Fundamentada en Datos) en la producción científica brasileña entre 2008-2012}

Maricel Karina López Torres, doutoranda em Engenharia e Gestão do Conhecimento na Universidade Federal de Santa Catarina (UFSC). E-mail: celdesigner@gmail.com.

Paulo Cristiano de Oliveira, doutorando em Engenharia e Gestão do Conhecimento na Universidade Federal de Santa Catarina (UFSC).

E-mail: oliveirapco@yahoo.com.br.

Carolina Schmitt Nunes, doutoranda em Engenharia e Gestão do Conhecimento na Universidade Federal de Santa Catarina (UFSC).

E-mail:nunes.carolinas@gmail.com.

Marina Keiko Nakayama, doutora em Administração pela Universidade Federal do Rio Grande do Sul e professora do Programa de Pós-Graduação em Engenharia e Gestão do Conhecimento da Universidade Federal de Santa Catarina (UFSC). E-mail:marina@egc.ufsc.br. 


\section{Resumo}

Este artigo tem como objetivo apresentar aspectos conceituais da Grounded Theorye identificar o delineamento das teses e dissertações brasileiras entre os anos de 2008 e 2012, em relação às três principais linhas ou escolas teóricas assumidas pelo método. Buscou-se identificar a abordagem clássica, a abordagem descritiva conceitual completa ( full conceptual description) e a abordagem construtivista, bem como as áreas ou temáticas trabalhadas no contexto da produção científica brasileira. Os procedimentos metodológicos incluíram a busca, a recuperação e a análise de documentos provenientes da Biblioteca Digital Brasileira de Teses e Dissertações (BDTD). Foram encontrados 278 documentos no período. Do total recuperado no sistema de busca, foram analisadas 182 teses e dissertações. Os resultados apontaram ênfase na utilização da Grounded Theorynas áreas de Enfermagem, Administração e Psicologia. Também se observou que os autores mais referenciados são Strauss e Corbin, seguidos por Charmaz, ou seja, há ênfase na full conceptual description e na abordagem construtivista. Identificada a utilização da Grounded Theory na produção de dissertações e de teses no contexto brasileiro, parece relevante refletir sobre a necessidade de promoção de formas de audiência e discussão sobre a sua prática em diferentes contextos, a fim de que ela possa alcançar o que se propõe na construção de conhecimento novo, em pesquisas.

Palavras-chave: Grounded Theory. Teoria Fundamentada. Escolas Teóricas. Produção Científica.

\section{Abstract}

This article aims to present the conceptual aspects of the Grounded Theory and identify the delineation of thesis and dissertations in Brazil between 2008 and 2012, in relation to the three main lines or theoretical schools assumed by the method. We tried to identify the classical approach, the full conceptual description approach, and the constructivist approach, as well as areas or themes addressed within the context of Brazilian scientific production. The methodological procedures included the search, recovery and analysis of documents from the Brazilian Digital Library of Thesis and Dissertations (BDTD). A total of 278 documents were found in this period. Of those recovered in the search 
system, we analyzed 182 thesis and dissertations. The results indicated emphasis on the use of Grounded Theory in the fields of nursing, administration and psychology. We also noted that the most frequently mentioned authors are Strauss and Corbin, followed by Charmaz. In other words, there is an emphasis on full conceptual description and on the constructivist approach. The adoption of Grounded Theory in the production of dissertations and thesis in the Brazilian context makes it relevant to reflect on the need to promote forms of audience and discussion about the theory's practice in different contexts, so that it can achieve what is proposed in the construction of new knowledge via research.

Keywords: Grounded Theory. Theoretical Schools. Scientific Production.

\section{Resumen}

Este artículo tiene como objetivo presentar aspectos conceptuales de la Grounded Theory y identificar el delineación de tesis científicas y disertaciones brasileñas entre los años 2008 y 2012, respecto a las tres principales líneas o escuelas teóricas asumidas por el método. Se trató de identificar los enfoques: clásico, descriptivo conceptual completo (full conceptual description) y constructivista, así como las áreas o temáticas adoptadas en el contexto de la producción científica brasileña. Los procedimientos metodológicos incluyeron la búsqueda, la recuperación y el análisis de documentos provenientes de la Biblioteca Digital Brasileira de Teses e Dissertações (BDTD). Se han encontrado 278 documentos en el período. Del total recuperado en el sistema de búsqueda, se han analizado182 tesis científicas y disertaciones. Los resultados indicaron énfasis de adopción de la Grounded Theory en el área de Enfermería, Administración y Psicología. Igualmente, se pudo observar que los autores más citados fueron Strauss y Corbin, seguidos por Charmaz, o sea, hay énfasis en el enfoque full conceptual description y en el constructivista. Identificada la adopción de la Grounded Theory en la producción de disertaciones y tesis en el contexto brasileño, parece relevante reflexionar acerca de la necesidad de promoción de formas de participación y de discusión acerca de su práctica en distintos contextos, con el fin de poder alcanzar lo que se propone en la construcción de nuevo conocimiento en investigaciones.

Palabras clave: Grounded Theory. Escuelas Teóricas. Producción Científica. 


\section{Introdução}

A realização deste trabalho se justifica na medida em que se entende oportuno divulgar e discutir a Grounded Theory (ou Teoria Fundamentada nos Dados), como uma abordagem de pesquisa qualitativa (NICO et al., 2007). Essa abordagem permite inúmeras possibilidades no âmbito de áreas diversas, como Administração (HOPFER; MACIEL-LIMA, 2008), Enfermagem (DANTAS et al., 2009) e Odontologia (NICO et al., 2007), por exemplo.

De acordo com Dantas et al. (2009), a Grounded Theory busca compreender a realidade a partir da percepção ou significado que certo contexto ou objeto tem para o indivíduo, gerando conhecimentos, aumentando a compreensão e proporcionando um guia significativo para a ação. Compreende uma metodologia qualitativa de investigação que extrai aspectos significativos das experiências vivenciadas pelos atores sociais, possibilitando interligar constructos teóricos e potencializando a expansão do conhecimento. No método, a coleta de dados, a análise e a eventual teoria mantêm uma relação próxima entre si. Um pesquisador não começa um projeto com uma teoria preconcebida em mente. Ao contrário, começa com uma área de estudo e permite que a teoria surja dos dados. De acordo com Cassiani, Caliri e Pelá (1996), a teoria fundamentada nos dados é vislumbrada como uma linha metodológica que pode ser utilizada em pesquisas interpretativas, uma vez que suas raízes estão ligadas ao interacionismo simbólico. Cabe observar que, embora o método possibilite a geração de uma teoria com base nos dados, ele também pode ser utilizado como instrumento norteador para a coleta, a análise e a interpretação dos dados.

Ante o exposto, o artigo tem como objetivo principal analisar teses e dissertações brasileiras disponíveis na Biblioteca Digital Brasileira de Teses e Dissertações (BDTD), defendidas entre os anos de 2008 e 2012, em relação às três principais linhas ou escolas teóricas assumidas pelo método da Grounded Theory, como proposto por Tarozzi (2011), ou seja, a abordagem clássica, a abordagem descritiva conceitual completa e a abordagem construtivista, bem como as áreas ou temáticas trabalhadas. A seguir, são apresentados o referencial teórico, os procedimentos metodológicos, os resultados, a discussão e as considerações finais. 


\section{Referencial teórico}

No referencial teórico deste artigo, é apresentado o conceito de Grounded Theory, sua descrição e suas principais características, os possivveis campos de aplicação e, por fim, as principais abordagens e pressupostos para sua utilização.

Segundo Strauss e Corbin (2008), a descoberta é o alvo da ciência desde o início da Renascença, entretanto, a maneira como a descoberta é feita varia de acordo com a natureza dos materiais estudados e com a época do estudo. Santos (2002), por sua vez, explica que, ao se fazer uma revisão da discussão sobre paradigmas nas ciências sociais, inevitavelmente se chegará à identificação de duas posições epistemológicas opostas - a positivista ou quantitativa e a fenomenológica ou interpretacionista.

A posição positivista ou quantitativa norteia-se pelo ideal regulador da física social, partindo do pressuposto de que as ciências naturais são aplicações ou concretizações de um modelo conhecido e universalmente válido. Sob essa ótica, é possível estudar os fenômenos sociais como fenômenos naturais, por maiores que sejam as diferenças entre eles. A outra posição, denominada fenomenológica ou interpretacionista, defende a ideia de que a ação humana é subjetiva. Nessa visão, o comportamento humano não pode ser descrito e tampouco explicado com base em suas características exteriores e objetiváveis (SANTOS, 2002). Para os interpretacionistas, a realidade social é, então, uma rede de representações complexas e subjetivas (VERGARA; CALDAS, 2005).

Em 1967, Barley Glaser e Anselm Strauss publicaram o volume intitulado The Discovery of Grounded Theory, no qual havia a primeira definição e formulação de um novo método para a pesquisa qualitativa. Na introdução, o texto continha uma definição bastante sintética da abordagem, dizendo que a "Grounded Theory é um método geral de análise comparativa [...] e um conjunto de procedimentos capazes de gerar [sistematicamente] uma teoria fundada em dados" (TAROZZI, 2011, p. 17, grifo do autor). 
Segundo Tarozzi (2011), desde a primeira definição, houve uma sucessão de percepções e opiniões sobre como uma Grounded Theory deve ser entendida e sobre sua classificação entre métodos e metodologias. Ele apresenta que, para Glaser (2004), a Grounded Theory é essencialmente uma metodologia. Para Strauss e Corbin (2008), é um método. Para Charmaz (2009), a Grounded Theory é uma constelação de métodos. Para Tarozzi (2011), a Grounded Theory pode ser entendida como uma metodologia que contém várias indicações de procedimentos, que assumem diversas declinações segundo a escola e os autores interessados. De maneira geral, a Grounded Theory pode ser considerada tanto um olhar teórico sobre o reconhecimento e a análise de dados, ou seja, um método geral, como também um conjunto de procedimentos e instrumentos concretos para recolher e analisar dados. Para os pesquisadores que se propõem a utilizar a abordagem Grounded Theory, são primordiais o conhecimento desses dois estágios e a consciência do nível de abstração necessário em cada um deles.

Uma pesquisa conduzida sob a ótica da Grounded Theory tem êxito se gerar uma teoria, ou seja, uma interpretação racional, articulada, sistemática e densa, capaz de dar conta da realidade estudada. A Grounded Theory tem a ambição de produzir uma teoria complexa e articulada, sublinhando com força a ligação íntima entre pesquisa teórica e pesquisa empírica e se colocando no espaço que une teoria e realidade (TAROZZI, 2011).

Strauss e Corbin (2008) afirmam que a Grounded Theorytende a se parecer mais com a realidade do que a teoria derivada da reunião de uma série de conceitos baseados em experiência ou somente por meio de especulação, em que se raciocina sobre como algo deveria funcionar. Para eles, o valor da Grounded Theory está em sua capacidade não apenas de gerar teoria, mas também de basear essa teoria em dados. Teorias fundamentadas, por serem baseadas em dados, tendem a oferecer mais discernimento, melhorar o entendimento e fornecer um guia importante para a ação (TAROZZI, 2011).

Tanto a teoria como a análise de dados envolvem interpretação, mas compreendem interpretação baseada em investigação feita 
sistematicamente. Se o objetivo do pesquisador for criar entendimentos novos e teoricamente expressos, então, os métodos de construção de teorias são fortemente indicados. Para tanto, são necessários e indicados diferentes processos de codificação de dados que permitam extrair categorias conceituais (STRAUSS; CORBIN, 2008; TAROZZI, 2011).

Em resumo, de acordo com Strauss e Corbin (2008), os procedimentos de codificação envolvem: 1) construir em vez de testar a teoria, 2) fornecer aos pesquisadores ferramentas analíticas para lidar com as massas de dados brutos, 3) ajudar os analistas a considerar significados alternativos para os fenômenos, 4) ser sistemático e criativo simultaneamente e 5) identificar, desenvolver e relacionar os conceitos que são os blocos de construção da teoria.

Para Cassiani, Caliri e Pelá (1996), a Grounded Theory é, sem dúvida, um processo complexo de análise de dados. É também um referencial de análise que fornece caminhos e orienta o investigador que pretende utilizá-la, tornando-se um norte metodológico para estudos interacionistas. No desenvolvimento da Teoria Fundamentada nos Dados, procura-se obter a complexidade e o movimento que só é possível no mundo real, embora se reconheça a incapacidade de visualizá-lo totalmente. Essa ligação íntima com os eventos e pessoas, portanto, talvez seja uma das grandes contribuições da abordagem interpretativa para a pesquisa social.

De acordo com Tarozzi (2011), considerando que existe pouca clareza sobre a utilização da Grounded Theory, só se pode falar dela se a pesquisa contemplar todas as características apresentadas no Quadro 1. Em casos em que a pesquisa não apresenta todas as características, tem-se outro tipo de estudo, possivelmente sem tanta ênfase nos dados empíricos coletados e que poderá resultar ou não em uma teoria. 


\section{Quadro 1. Principais características da Grounded Theory}

\begin{tabular}{|c|c|}
\hline Característica & Descrição \\
\hline $\begin{array}{l}\text { Exploração de um } \\
\text { processo }\end{array}$ & $\begin{array}{l}\text { Mesmo partindo da linguagem e dos significados, a } \\
\text { Grounded Theory busca regularidade de tipo conceitual } \\
\text { entre os fenômenos a serem analisados. Tem por fim fazer } \\
\text { emergir os processos sociais e os processos psicológicos } \\
\text { de base que subjazem aos fenômenos indagados. Procura } \\
\text { explorar processos subjacentes aos fenômenos estáticos } \\
\text { e suas dinâmicas. }\end{array}$ \\
\hline Amostragem teórica & $\begin{array}{l}\text { É uma função do processo analítico e se apresenta como } \\
\text { uma extensão progressiva, no decorrer da análise, do } \\
\text { número e das características dos participantes, uma } \\
\text { extensão conduzida pelas exigências do trabalho de } \\
\text { conceituação teórica. A amostra não se forma a priori, mas } \\
\text { no decorrer da pesquisa, seguindo as lacunas da teoria } \\
\text { emergente, para chegar a saturar as categorias. }\end{array}$ \\
\hline $\begin{array}{l}\text { Simultaneidade entre } \\
\text { coletar e analisar os } \\
\text { dados }\end{array}$ & $\begin{array}{l}\text { Acompanhamento constante da reflexão analítica } \\
\text { com periódicos retornos ao campo onde o processo de } \\
\text { recolhimento dos dados é guiado pela reflexão analítica } \\
\text { sobre as categorias emergentes. Esse é um dos traços mais } \\
\text { desafiadores, porque se predispor à coleta de dados, tendo } \\
\text { a dimensão analítica em paralelo, é uma operação complexa } \\
\text { e talvez pouco natural. }\end{array}$ \\
\hline $\begin{array}{l}\text { Uso do método da } \\
\text { constante comparação } \\
\text { em todos os níveis de } \\
\text { análise }\end{array}$ & $\begin{array}{l}\text { É um método geral para a investigação, no qual se } \\
\text { confrontam constantemente dados entre si, etiquetas } \\
\text { geradas na primeira codificação, diferentes eventos } \\
\text { observados e as categorias entre si. Esse aspecto convida } \\
\text { a apresentar sempre perguntas aos dados, nos vários níveis } \\
\text { de análise. Essas perguntas buscam nexos entre dados } \\
\text { e conceitos, favorecendo o progresso da compreensão } \\
\text { conceitual dos fenômenos estudados. }\end{array}$ \\
\hline $\begin{array}{l}\text { Construção de uma } \\
\text { codificação por meio } \\
\text { dos dados }\end{array}$ & $\begin{array}{l}\text { Mantém e preserva um forte enraizamento da conceituação } \\
\text { nos dados empíricos. A maneira gradual e progressiva de } \\
\text { codificar é necessária para manter a evidência e os traços } \\
\text { do percurso gerativo dos conceitos que constituem a teoria } \\
\text { enraizada. }\end{array}$ \\
\hline $\begin{array}{l}\text { Elaboração de } \\
\text { conceitos }\end{array}$ & $\begin{array}{l}\text { Busca a elaboração conceitual e não a descrição, como } \\
\text { exigido pela maior parte dos demais métodos. As análises } \\
\text { que não conseguem decolar do plano da descrição, por } \\
\text { mais acuradas e sistemáticas que sejam, não podem ser } \\
\text { consideradas como Grounded Theory. }\end{array}$ \\
\hline $\begin{array}{l}\text { Produção de } \\
\text { memorandos e de } \\
\text { diagramas }\end{array}$ & $\begin{array}{l}\text { Toda Grounded Theory deve apresentar alguns arcabouços } \\
\text { instrumentais típicos que respondem às exigências } \\
\text { metodológicas específicas do método. Portanto, anotações } \\
\text { sobre o processo de pesquisa e diagramas, como mapas } \\
\text { conceituais, funcionam como andaimes nos níveis } \\
\text { superiores de elaboração teórica, quando se trata de } \\
\text { acompanhar a constante comparação entre as categorias } \\
\text { e as macrocategorias já bastante abstratas. }\end{array}$ \\
\hline
\end{tabular}

Fonte: Elaboração dos autores. Adaptado de Tarozzi (2011, p. 21-28). 
A Grounded Theoryse contrapõe ao método hipotético-dedutivo de pesquisa. Segundo Hughes (1983), o empirismo puro não pode gerar leis científicas universais, que só seriam obtidas por meio da lógica segundo a qual o determinismo e a necessidade são consequência de uma estrutura formal. De premissas gerais, se as regras dedutivas fossem obedecidas de maneira correta, deveria ser obtida uma conclusão de argumento lógico.

De acordo com Tarozzi (2011), a teoria enraizada propõe um método também empírico para produzir rigorosamente uma teoria por intermédio de uma abordagem indutiva ou por abdução. 0 método de relacionar conceitos dentro de proposições sintéticas, as únicas proposições consideradas relevantes para a ciência, é denominado por ele de indução (HUGHES, 1983, p. 51).

A abdução, teorizada por Peirce (1984), é um raciocínio rigoroso e probabilístico, que parte de uma premissa certa, mas que é criativo e não tautológico, porque sua premissa menor é só provável. Nessa probabilidade, existe espaço para a descoberta do novo, do insondável, do não conhecido. Para que seja acionado esse pensamento, é necessário um evento fortuito, um êxito inesperado, um episódio iluminador. E, em seguida, faz-se premente um elevado espírito de observação que permita olhar para penetrar a fundo nos fenômenos - em todos os fenômenos.

Ainda segundo Peirce (1984), a abdução é o processo para formar hipóteses explicativas. A dedução prova algo que deve ser; a indução, por sua vez, mostra algo que atualmente é operatório. A abdução faz uma mera sugestão de algo que pode ser. Portanto, para apreender ou compreender os fenômenos, só a abdução pode funcionar como método. Assim, a Grounded Theorypropõe soluções não verdadeiras e abstratas, mas corretas e certas, altamente prováveis e concretas, a partir até mesmo de um único caso. Ela não se limita a recolher dados e analisálos para verificar ou falsificar teorias preexistentes, pensadas em outro contexto e por outras pessoas, mas, utilizando-se dos dados, constrói criativa e rigorosamente uma teoria capaz de explicar os fenômenos estudados (TAROZZI, 2011). 
Cabe destacar que a Grounded Theory tem sido associada a diferentes áreas de conhecimento, como, por exemplo, as de Administração e da Saúde. Hopfer e Maciel-Lima (2008) conduziram um estudo que buscou realizar uma avalição crítica do método nos estudos organizacionais. Os autores realizaram um rastreamento na base de dados Business Source Elite (BSE), no Portal da Coordenação de Aperfeiçoamento de Pessoal de Nivel Superior (Capes) e nos Anais do Encontro Nacional da Associação de Pós-Graduação e Pesquisa em Administração (Enanpad), de artigos publicados entre 2000 e 2006 . Foram encontrados artigos com pesquisa específica na área de Administração apenas a partir de 2006. Os artigos analisados utilizam a Grounded Theory geralmente associada a outros métodos de pesquisa e, assim, não seguiram todas as etapas previstas pelo método. Na visão desses autores, a Grounded Theory mostrou-se uma abordagem intersubjetiva, pois, mesmo iniciando no extremo subjetivismo da fase inicial de Glaser e Strauss, as etapas do método levaram a uma tentativa de objetivação dos dados para dar conta de construir uma explicação do fenômeno.

Nico et al. (2007), por sua vez, discutiram a utilização da Grounded Theory na área da Saúde, especificamente na Odontologia. Segundo eles, as abordagens qualitativas estão sendo mais empregadas pelos pesquisadores da área. Essa abordagem metodológica torna-se fundamental, no sentido de propiciar a exploração de assuntos ainda pouco trabalhados nessa perspectiva metodológica, possibilitando que as pesquisas adquiram contribuições originais e, assim, ampliando o conhecimento na área. Ainda com foco na Saúde, Silva et al. (2011) elaboraram estudo que buscou identificar as teses e dissertações defendidas nos programas de pós-graduação em Enfermagem do Brasil que utilizaram o referencial metodológico da Grounded Theory e analisaram os aspectos epistemológicos. Os dados foram coletados no Banco de Teses da Capes, no recorte temporal entre 1996 e 2010. Foram levantados 99 resumos, e as áreas temáticas mais expressivas foram: Saúde da Criança/Adolescente; Gestão em Saúde/Enfermagem; Saúde Coletiva e Saúde da Mulher. O principal referencial teórico utilizado foi o interacionismo simbólico. Para as autoras, a Grounded Theory compreende uma metodologia que pode ser empregada na Enfermagem e que é capaz de contribuir no desenvolvimento de novas investigações para o seu aprimoramento contínuo, o que corrobora a visão de Nico et al. (2007). 
Com relação às principais abordagens, constata-se que, nos primeiros textos relacionados à Grounded Theory, não houve uma dedicação especial sobre o panorama epistemológico no qual a nova teoria se inseria. De modo especial, a Grounded Theoryé apresentada como uma abordagem metodológica que nasce da prática de pesquisa. A falta de uma epistemologia explícita de referência pode representar um limite, e historicamente foi isso o que aconteceu. Sem epistemologia de referência, a Grounded Theory foi, no decorrer do tempo, objeto de muitos debates de adeptos de diferentes abordagens, apoiando-se em diferentes paradigmas de pesquisa, como o sociológico quantitativo, o pragmatismo, o interacionismo simbólico e a fenomenologia. Esta última, embora não represente um antecedente direto, poderia constituir um referencial teórico e um panorama epistemológico sólido e atual (TAROZZI, 2011).

Em face do conflito de visão metodológica que se criou entre os dois fundadores da Grounded Theory, delineiam-se, conforme Tarozzi (2011), três principais linhas ou escolas que o método assumiu nos últimos anos:

- Na abordagem clássica, influenciada por Glaser (2004), a pergunta de pesquisa não é uma afirmação que identifica o problema a ser pesquisado, pois o autor entende que é impossível definir o problema antes de ir para o campo. Iniciase a pesquisa de modo aberto, em uma área de investigação. Todos os dados são considerados, e a categoria central (core category) emerge quase que magicamente, sendo intuída improvisadamente no início ou no fim de uma pesquisa. 0 tipo de codificação utilizada é a substantiva teórica.

- Na abordagem descritiva conceitual completa, influenciada por Strauss e Corbin (2008), a pergunta de pesquisa é uma afirmação que identifica claramente o problema a ser estudado, conseguindo restringir e gerenciar a área de investigação. Não há diferença quanto ao tipo de dados utilizados, privilegiando-se, acima de tudo, as observações. Para fazer com que emerja a core category, que não é única, é necessária intensa manipulação de dados. A codificação é aberta, axial e seletiva. 
- Na abordagem construtivista, influenciada por Charmaz (2009), não existe uma pergunta de pesquisa. Existe a ideia de conceitos sensibilizantes, interesses pessoais e disciplinares que iniciam a pesquisa. Como tipos de dados utilizados para a pesquisa, sugerem-se entrevistas semiestruturadas, análise textual e coconstrução de dados, partindo-se do pressuposto de que existe uma core category prevalente. A codificação é inicial, focalizada, axial e teórica.

As três abordagens ou articulações que o método assumiu nos últimos 40 anos podem levar à construção de uma teoria substantiva. São caminhos distintos para o mesmo objetivo central. Cabe ao pesquisador escolher que caminhos utilizará no seu percurso metodológico, sendo que a literatura não estabelece se haveria uma abordagem mais correta do que as demais (TAROZZI, 2011). Os princípios norteadores da pergunta de pesquisa, os tipos de dados, a core categorye os tipos de codificação de cada uma das abordagens devem ser respeitados, para que se possa garantir coerência no decorrer da pesquisa.

\section{Procedimentos metodológicos}

Este estudo se consistiu na recuperação e na análise de teses e dissertações provenientes de instituições de ensino e pesquisa, disponíveis em meio eletrônico no repositório da Biblioteca Digital Brasileira de Teses e Dissertações - BDTD (SOUTHWICK, 2003). Trata-se de pesquisa exploratória, na medida em que proporciona uma visão geral do uso da Grounded Theory no desenvolvimento de estudos relatados em teses e dissertações (GIL, 2008). Aqui, buscou-se identificar as três principais escolas da Grounded Theory e seus respectivos autores, descritas por Tarozzi (2011): GT clássica (Glaser), GT full conception description (Strauss e Corbin) e GT construtivista (Charmaz)1.

Segundo as informações apresentadas pela própria biblioteca, ela conta com a parceria de 97 universidades desde o ano 2000 e continha um total de 193.938 teses e dissertações na época em que a busca foi

${ }^{1}$ A pesquisa foi realizada no período entre maio e junho de 2012. realizada (BDTD, 2010a). O total de documentos registrados na série histórica, por ano, está representado na Figura 1 (BDTD, 2010c). 


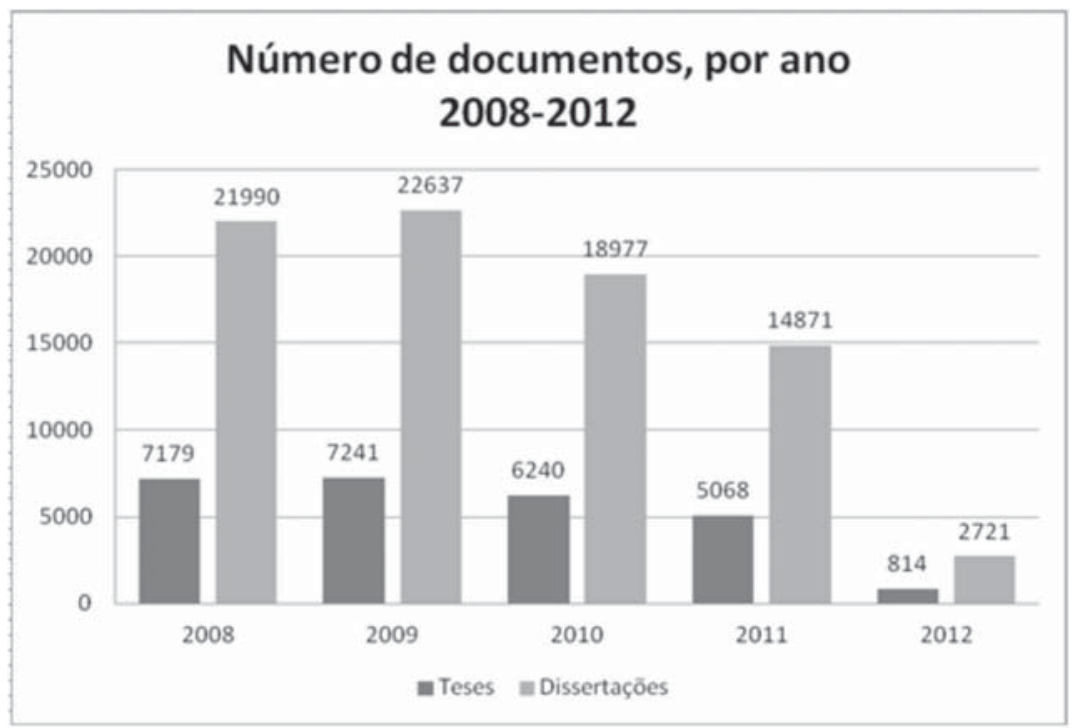

Fonte: Elaboração dos autores.

Figura 1. Número de documentos disponíveis na BDTD, no período entre janeiro de 2008 e junho de 2012

Os documentos provenientes da BDTD constituíram a principal fonte de dados para a análise, apresentando vantagens e desvantagens (CRESWELL, 2010). Tal escolha se deu em razão de vantagens, como: a) a possibilidade de obter a linguagem e as palavras utilizadas pelos pesquisadores brasileiros; b) a facilidade de acesso - a maior parte das teses e dissertações estava disponivel on-line; c) a apresentação de conteúdo elaborado de forma criteriosa; e d) a facilidade de organização e de transcrição dos dados. Entre as desvantagens, destacam-se a indisponibilidade de algumas teses e dissertações (por solicitação de patente) e a limitação das alternativas de refinamento do sistema de busca. Além dos documentos, a bibliografia sobre a Grounded Theory também foi considerada como fonte de dados, pois contém informações relevantes sobre o método - sobretudo no que se refere a suas características e abordagens. É importante salientar que os documentos referentes ao ano de 2012 ainda estavam em fase de inclusão na base de dados e, portanto, não constituíam dados definitivos (foram considerados dados até junho de 2012). 


\section{Primeiros passos para a coleta de dados}

Para realizar a coleta de dados, optou-se por procura básica na BDTD utilizando o termo Grounded Theory, o que resultou em 278 documentos encontrados no período 2001 a 2012. Do total recuperado no sistema de busca, foram analisadas as obras publicadas nos últimos cinco anos, disponíveis em meio eletrônico, totalizando 183 teses e dissertações. $\mathrm{O}$ total de documentos analisados garantiu uma amostra representativa da base de dados².

Desse total de documentos (278), a pesquisa retornou 183 resultados para a busca do termo Grounded Theory, sendo que 52,5\% ( $n=96$ ) das teses e dissertações correspondiam a pesquisas qualitativas ou de métodos mistos, embora sem fazer uso da Grounded Theory como abordagem metodológica. Esses 52,5\%, mais 2,2\% ( $n=4)$ que não puderam ser analisados porque o arquivo não estava disponível, juntamente com o 0,5\% (n=1) que correspondia ao ano 2007, foram desconsiderados na análise.

Portanto, 44,8\% (n=82) dos documentos foram analisados para verificar a abordagem da GT empregada na pesquisa. Desse total, prevaleceu o seu uso em dissertações, sendo $40,2 \%(n=33)$ de teses e $59,8 \%(n=49)$ de dissertações - distribuição essa que pode ser observada na Figura 2.

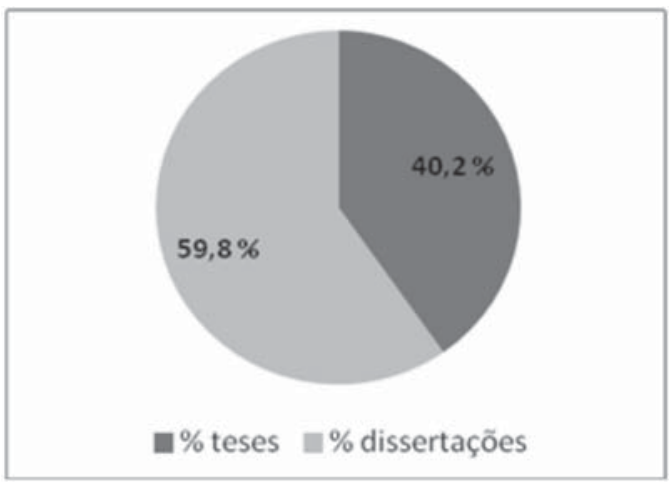

2 A amostra analisada representa 5\% de erro amostral, com nível de confiança entre 95\% e 99\% - sendo $\mathrm{n}=162$, para $95 \%$, e $\mathrm{n}=197$, Fonte: Elaboração dos autores.

Figura 2. Distribuição da frequência de teses e dissertações analisadas 
Para proceder à análise do conteúdo desses documentos (CRESWELL, 2010), os dados foram compilados em planilha eletrônica e em arquivo de texto. Os principais dados reunidos na planilha eletrônica foram: o ano da defesa, o tipo de produção (tese/dissertação), o título, a área de estudo, a abordagem/autores da Grounded Theorye outros que poderão ser objeto de estudos posteriores, como bases epistemológicas, instrumentos e técnicas de coleta de dados adotadas. Os resumos de cada uma das teses e dissertações coletadas foram reunidos em um arquivo de texto para a análise das palavras ali contidas.

\section{Registro e análise das informações}

Os dados coletados foram tabulados, codificados, apresentados em percentuais e representados por elementos visuais, na forma de gráficos e tabelas. Para complementar a análise de frequência das diferentes categorias, foi realizada a verificação da ocorrência de palavras nos resumos, elaborando nuvens de palavras com o uso do aplicativo TagCrowd, o que possibilitou a visualização da frequência com que as palavras aparecem em um texto, como forma de análise qualitativa (STEINBOCK, 2012; CIDELL, 2010). Tal técnica é denominada análise de conteúdo (TRIVIÑOS, 2011), na perspectiva de análise alternativa à tradicional, tratando “da sistematização, da tentativa de conferir maior objetividade a uma atitude que conta com exemplos dispersos, mas variados, de pesquisa com textos" (ROCHA; DEUSDARÁ, 2005, p. 308).

Com base no documento contendo o agrupamento de palavras dos resumos, o aplicativo analisou 4.577 palavras possíveis, de um total de 27.467 palavras. Para a análise, foram utilizados os parâmetros: idioma Português, visualização de 100, 50 e 25 palavras, com e sem visualização da contagem, sucessivamente. Ao selecionar o idioma, o aplicativo suprimiu os acentos e não analisou palavras comuns preposições e afins, denominadas stop words.

Antes da análise, a acentuação, o gênero (masculino/feminino), o número (singular/plural), o tempo verbal e a sinonímia das palavras foram igualados para proporcionar maior confiabilidade ao resultado. 
Isso foi feito sucessivamente e quando necessário. A sinonímia foi utilizada somente quando se referiam a um mesmo ser no texto e havendo coesão lexical, como apresentado por Seidi (2008). Os resultados das análises e a discussão são apresentados a seguir.

\section{Resultados e Discussão}

Nesta seção são apresentados: o uso da Grounded Theory na produção acadêmica analisada, a distribuição de uso por área temática, as instituições que originaram essas produções, a abordagem da Grounded Theory adotada pelos autores das teses e dissertações e o processo investigativo empregado.

Na Figura 3 é possível visualizar o número de teses ou dissertações que adotaram a Grounded Theory na sua abordagem metodológica (por ano), sendo maior no ano de 2009 (dissertações, n=17; teses, $n=10)$. Os motivos que levaram a essa evidência não foram objeto deste estudo.

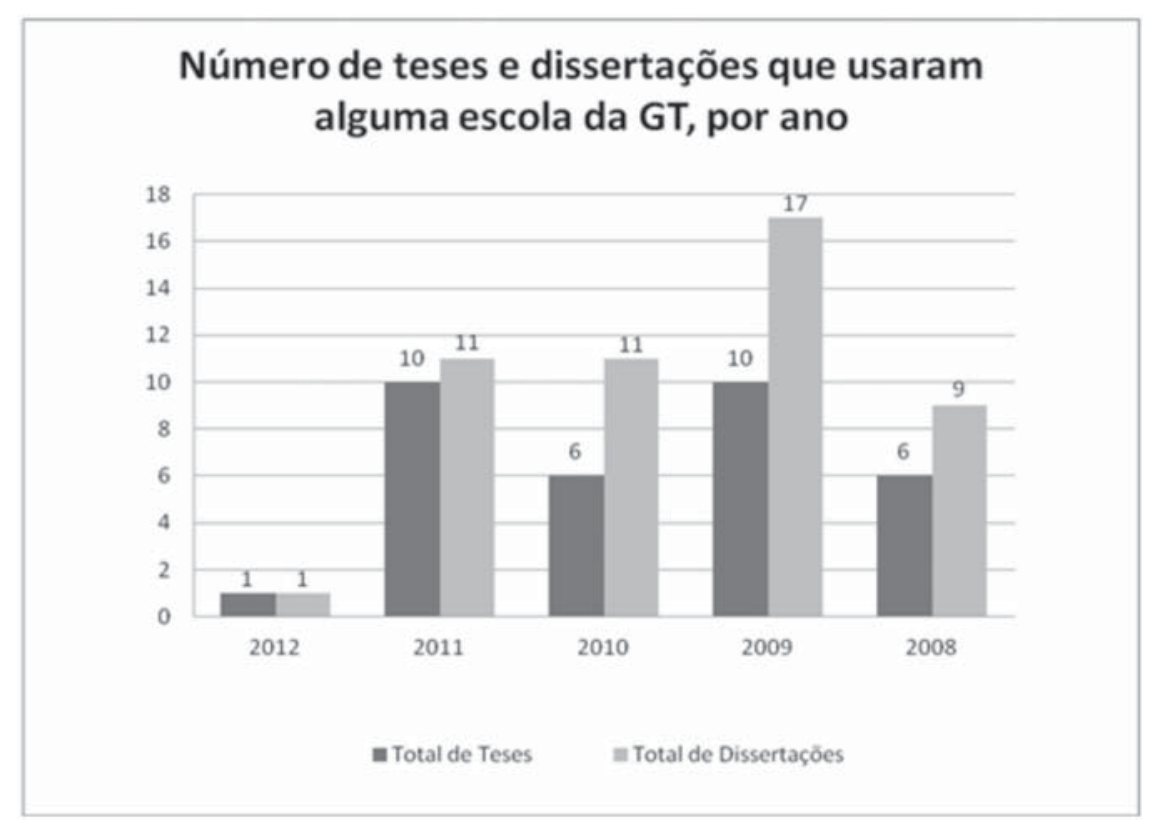

Fonte: Elaboração dos autores.

Figura 3. Uso da Grounded Theory em teses e dissertações 
No que se refere às áreas temáticas em que alguma abordagem da Grounded Theory foi empregada, despontam a Enfermagem ( $n=20$ ), a Administração $(n=18)$ e a Psicologia $(n=10)$, seguidas da Educação $(n=7)$ e Ciências Ambientais e da Saúde (n=6). Essas áreas temáticas foram obtidas analisando-se o curso e o programa de pós-graduação ao qual pertenciam. Outras áreas menos frequentes também podem ser visualizadas na Tabela 1.

Tabela 1. Distribuição de uso da Grounded Theory por área temática

\begin{tabular}{l|c|c}
\hline \multicolumn{1}{c|}{ Áreas Temáticas } & $\mathbf{n}$ & $\%$ \\
\hline $\begin{array}{l}\text { Enfermagem I Enfermagem Psiquiátrica | } \\
\text { Enfermagem, Saúde e Sociedade }\end{array}$ & 20 & 24,4 \\
\hline Administração | Administração de Empresas & 18 & 22,0 \\
\hline Psicologia | Psicologia Aplicada & 10 & 12,2 \\
\hline Educação | Educação Ambiental & 7 & 8,5 \\
\hline Ciências Ambientais e da Saúde & 6 & 7,3 \\
\hline Ciência da Informação & 3 & 3,7 \\
\hline Engenharia Civil I Engenharia de Produção & 3 & 3,7 \\
\hline Ciência da Computação & 2 & 2,4 \\
\hline Estudos da Linguagem I Linguística & 2 & 2,4 \\
\hline Informática I Informática na Educação & 2 & 2,4 \\
\hline Arquitetura e Urbanismo & 1 & 1,2 \\
\hline Ciências Contábeis & 1 & 1,2 \\
\hline Ciências Odontológicas & 1 & 1,2 \\
\hline Ciência & 1 & 1,2 \\
\hline Gestão do Conhecimento e Tecnologia da Informação & 1 & 1,2 \\
\hline Letras & 1 & 1,2 \\
\hline Meios e Processos Audiovisuais & 1 & 1,2 \\
\hline Serviço Social & 1,2 \\
\hline Tecnologias da Inteligência e Design Digital & 1,2 \\
\hline
\end{tabular}

Fonte: Elaboraç̃o dos autores.

Na literatura, foram encontrados estudos de revisão como os de Hopfer e Maciel-Lima (2008), na área de Administração, enquanto Nico et al. (2007) e Silva et al. (2011) realizaram revisões sobre o emprego 
da Grounded Theory na área da Saúde, todas elas vindo ao encontro dos resultados obtidos. Entretanto, na área da Educação (Educação, Educação Ambiental) não foram encontrados estudos que fizessem uma revisão sobre o emprego da Grounded Theory em pesquisa nessa área.

A análise dos resumos do conjunto de teses e dissertações das cinco áreas de estudo que mais se destacaram (Enfermagem, Administração, Psicologia, Educação e Ciências Ambientais e Saúde) resultou na tagcloud(Figura 4) contendo 100 palavras, especialmente no que se refere à possibilidade de uso da GT para coletar dados referentes a indivíduos (por exemplo, enfermeiro; $n=25$ ou criança; $n=22$ ) ou a grupos (empresas; $n=39$ ), em um dado contexto ( $n=41)$. Na área da Saúde, o termo "criança" é um exemplo de temática mais expressiva, conforme o estudo de Silva et al. (2011). Pode-se inferir que tais palavras denotam ligação íntima com eventos e pessoas em um dado contexto explicitado pelos pesquisadores, vindo ao encontro do proposto por Cassiani, Caliri e Pelá (1996).

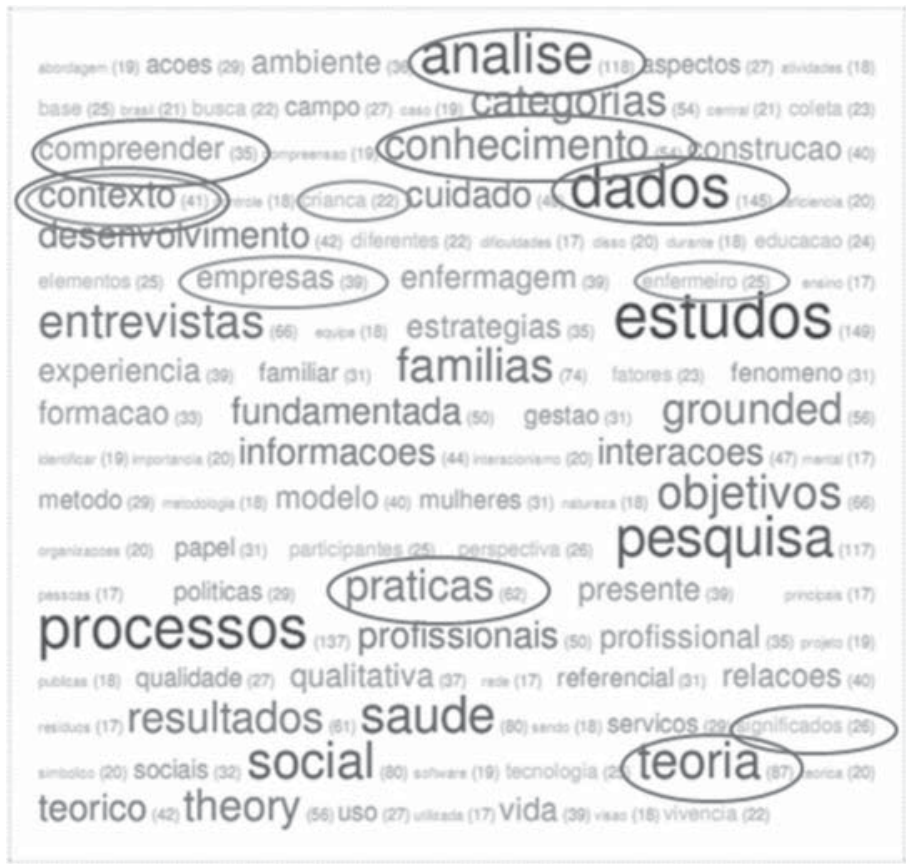

Fonte: Elaboração dos autores.

Figura 4. Tagcloud contendo 100 palavras, elaborada com o aplicativo TagCrowd 
Em particular (Figura 4), evidencia-se a relação da Grounded Theorycom estudos de diferentes naturezas envolvendo análise (n=118), forte relação com dados ( $n=145$ ) e também enquanto teoria ( $n=87$ ). Identifica-se ainda a relação com as práticas ( $n=62)$, como proposto por Vergara e Caldas (2005), e com o contexto ( $n=41)$, com a finalidade de compreender $(n=35)$ e atribuir significado $(n=26)$ ao conhecimento ( $n=54)$. Mais uma vez, os dados vêm ao encontro do proposto por Dantas et al. (2009).

Com relação às instituições de ensino superior às quais os trabalhos estão vinculados, observou-se uma predominância da Universidade de São Paulo (USP), com 17 trabalhos, como mostra a Tabela 2. Na sequência, vêm a Pontifícia Universidade Católica de Goiás, a Pontifícia Universidade Católica de São Paulo e a Universidade Federal do Rio Grande do Sul, cada qual com sete trabalhos. E, ainda com um número expressivo de trabalhos, as Universidades Federais da Paraíba e de Santa Catarina, cada qual com cinco trabalhos. Além das seis universidades apresentadas na Tabela 2, outras 18 instituições de ensino superior apresentaram trabalhos adotando a Grounded Theory.

\section{Tabela 2. Instituições de ensino de origem das teses e dissertações} (2008-2012)

\begin{tabular}{l|c|c}
\hline Universidade & $\mathbf{n}$ & $\%$ \\
\hline Universidade de São Paulo & 17 & 20,73 \\
\hline Pontifícia Universidade Católica de Goiás & 07 & 8,53 \\
\hline Pontifícia Universidade Católica de São Paulo & 07 & 8,53 \\
\hline Universidade Federal do Rio Grande do Sul & 07 & 8,53 \\
\hline Universidade Federal da Paraíba & 05 & 6,09 \\
\hline Universidade Federal de Santa Catarina & 05 & 6,09 \\
\hline
\end{tabular}

Fonte: Elaboração dos autores.

O número mais expressivo de trabalhos oriundos da Universidade de São Paulo pode ser um indicativo da existência de grupos de pesquisa dedicados ao emprego da Grounded Theory. Entretanto, a análise dessa vinculação não constitui objeto deste estudo, embora pareça relevante considerar a necessidade de articular e promover discussões sobre as abordagens, seus alcances e limitações, tanto no âmbito institucional, 
quanto no meio científico brasileiro como um todo. Tal feito seria válido para aprimorar e consolidar a prática da Grounded Theorye suas diferentes contribuições no campo da pesquisa.

No que se refere aos autores correspondentes a cada uma das abordagens que se investigou e que foram citados por Tarozzi (2011) - Strauss, Corbin e Charmaz, precursores da Grounded Theory -, identificou-se que a maioria dos estudos não deixava explícita a sua aderência à Grounded Theory clássica (Glaser), à Grounded Theory full conceptual description (Strauss e Corbin) ou à Grounded Theory construtivista (Charmaz). Ainda assim, identificou-se que a maioria das teses e dissertações referenciava Strauss e Corbin em seus procedimentos metodológicos (40,2\%; n=33), seguidos da citação de um mix de autores que incluía Charmaz, Corbin, Glaser e/ ou Strauss (34,1\%; $n=34,1)$ como pode ser observado na Tabela 3.

Tabela 3. Autores referenciados nas teses e dissertações (2008-2012)

\begin{tabular}{l|c|c}
\hline Grounded Theory & N & $\%$ \\
\hline Strauss e Corbin & 33 & 40,2 \\
\hline Charmaz & 12 & 14,6 \\
\hline Glaser e Strauss & 4 & 4,9 \\
\hline mix de autores & 28 & 34,1 \\
\hline outros & 5 & 6,1 \\
\hline
\end{tabular}

Fonte: Elaboração dos autores.

Embora Strauss e Corbin sejam os autores que influenciaram a abordagem full conceptual description (TAROZZI, 2011), não é possível afirmar que, em todas as teses e dissertações que os citaram, os autores seguiram tal abordagem e seus métodos, dado que muitos deles não aprofundam o esclarecimento desses aspectos nas pesquisas que realizaram em seus estudos. De forma geral, um número restrito de autores deixa clara a abordagem adotada, ao se considerar a classificação apresentada por Tarozzi (2011), limitando-se a descrever os passos incorporados ao processo de investigação. Além disso, assim como 
foi observado por Cassiani, Caliri e Pelá (1996) e Silva et al. (2011), muitos autores descrevem o uso da Grounded Theory associada ao interacionismo simbólico. Também se destaca a utilização da abordagem construtivista comentada por Tarozzi (2011).

Na Tabela 4 está demonstrada a preferência pelo uso da Grounded Theory para conduzir a pesquisa desde a coleta dos dados e em todo o processo investigativo (57\%; $n=47$ ), porém com pouca diferença em relação ao uso da Grounded Theory somente para a análise e interpretação dos dados gerados ou coletados mediante outras abordagens e/ ou técnicas (41,5\%; $n=34)$. Esse cenário pode indicar que os pesquisadores ainda não encontraram um caminho consensual para a condução dos procedimentos metodológicos de seus estudos utilizando a Grounded Theory. Para Strauss e Corbin (2008), um importante papel da Grounded Theory é a sua utilização como um conjunto de ferramentas ou técnicas para facilitar a análise de dados, que pode ser utilizado de forma flexível, para a construção de teorias criativas, fundamentadas e densas. Assim, a utilização da Grounded Theory somente para análise e interpretação dos dados não desqualificaria as teses e dissertações identificadas nas pesquisas que a adotaram como processo investigativo, mas seria um caminho possível para a condução das pesquisas, desde que a análise seja pautada por um dos referenciais de base e que estejam explicitados os procedimentos, as escolhas e as decisões de todo o processo de pesquisa.

Tabela 4. Processo investigativo adotado pelos autores, usando a Grounded Theory (2008-2012)

\begin{tabular}{l|c|c}
\hline Processo investigativo & $\mathbf{n}$ & $\%$ \\
\hline método geral - coleta, organização e análise de dados & 47 & 57,3 \\
\hline análise e interpretação de dados & 34 & 41,5 \\
\hline n. i. & 1 & 1,2 \\
\hline
\end{tabular}

Fonte: Elaboração dos autores.

Observa-se que, embora não tenham sido identificadas outras escolas, alguns estudos referenciam apenas trabalhos recentes que utilizaram alguma forma de abordagem denominada Grounded Theory 
por seus autores. Sendo a Grounded Theory uma abordagem que pode ser adaptada às diversas modalidades de pesquisa, compreende-se que se trata de uma forma de aplicação que, originalmente, pode ter sido embasada nas escolas descritas por Tarozzi (2011), porém sem adotar explicitamente essa classificação.

\section{Considerações Finais}

No decorrer do estudo, percebeu-se que a aplicação da Grounded Theory (Teoria Fundamentada nos Dados) tem ocorrido em diferentes áreas de conhecimento. A sua adoção em pesquisa brasileira tornou oportuna a discussão proposta por este artigo, uma vez que a referida teoria pode ser aplicada em diversas áreas e conduzida de diferentes formas.

Identificada a escolha da Grounded Theory para a produção tanto de dissertações quanto de teses no contexto brasileiro, parece relevante refletir sobre a promoção de formas para audiência e discussão sobre a sua prática em diferentes contextos, a fim de que ela possa alcançar o que se propõe na construção de conhecimento novo, em pesquisas. Tal feito poderia ocorrer tanto em eventos das mais diferentes áreas que vêm adotando o método quanto no interior ou entre grupos de pesquisa vinculados a cursos de pós-graduação.

Especialmente dada a complexidade envolvida na construção da teoria, é oportuno discutir sobre a necessidade de cautela quando da sua utilização em dissertações de mestrado, uma vez que o tempo para a saturação dos dados tende a ser significativamente menor do que em pesquisas de doutorado. Isso também conduz a um maior esclarecimento sobre o alcance da Grounded Theory, evitando sua adoção como mera técnica de coleta de dados qualitativos, mas, sobretudo, como análise criteriosa para a geração de teoria, desde a coleta e a análise até a saturação teórica.

Verificou-se também que as áreas temáticas que mais utilizaram a metodologia, foram a Enfermagem, a Administração, a Psicologia e a Educação, o que indica o caráter transdisciplinar da Grounded Theory. 
Percebe-se que ainda há inúmeras áreas que podem desenvolver estudos utilizando-a na condução de pesquisa (por exemplo, em áreas multidisciplinares ou em áreas interdisciplinares). Além da observada existência de trabalhos de revisão em áreas específicas, como a Administração e a Saúde, identificou-se a possibilidade de ampliar o escopo de revisão à Educação, área em que o uso da Grounded Theory ainda é incipiente e para a qual poderia contribuir com a geração de teorias contextualizadas e fortemente enraizadas nos dados coletados em campo.

Quanto à prática da Grounded Theory, com este estudo, identificou-se que os autores mais referenciados são Strauss e Corbin, seguidos de Charmaz. Tal evidência parece indicar ênfase na full conceptual description e na abordagem construtivista. Igualmente, chamou a atenção a utilização de um mix de autores para fazer referência à Grounded Theory. Parece-nos que seria relevante indicar a necessidade de um posicionamento mais objetivo por parte dos autores que adotam a Grounded Theoryem seus estudos, evitando abordagens que incluam autores com diferentes perspectivas - ou escolas, como apresentado por Tarozzi (2011) - sobre o seu uso ou, ainda, deixando clara a influência de cada uma dessas escolas no estudo proposto.

Para finalizar, no que se refere às limitações do estudo, cumpre esclarecer que este não buscou avaliar a coerência entre a escola e os métodos adotados por seus autores. Em outras palavras, não teve por objetivo avaliar a consistência de uso da escola e a base epistemológica adotada pelos pesquisadores. Dessa forma, sugere-se a realização de trabalhos voltados para tais questões, sem comprometer os resultados aqui apresentados. Inclusive, cumpre informar que não se trata de um estudo necessariamente representativo do total de instituições e cursos existentes em todo o Brasil, limitando-se àquelas que constituíram parceria com a BDTD até a época desta pesquisa, mas que propiciaram uma análise importante da produção científica brasileira entre 2008 e 2012 no que tange à utilização da Grounded Theory(Teoria Fundamentada nos Dados). 


\section{Referências}

BDTD - Biblioteca Digital Brasileira de Teses e Dissertações. Indicador resumo geral. Série histórica por Instituições de Defesa [resumo]. BDTD, 2010a. Disponivel em: <http://bdtdj.ibict.br/indicadores/graficoRS.

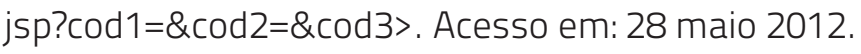

O que é a BDTD? BDTD, 2010b. Disponivel em: < http:/ / bdtd.ibict. br/pt/a-bdtd.html>. Acesso em: 20 maio 2012.

Série histórica consolidada por ano de defesa. BDTD, 2010c. Disponível em: <http://bdtdj.ibict.br/indicadores/graficoSHCAD. jsp?decada=2000>. Acesso em: 28 maio. 2012.

CASSIANI, S. de B.; CALIRI, M. H. L.; PELÁ, N. T. R. A teoria fundamentada nos dados como abordagem da pesquisa interpretativa. Revista Latinoamericana de Enfermagem, v. 4, n. 3, p. 75-88, 1996.

CHARMAZ. K. A construção da teoria fundamentada: guia prático para análise qualitativa. Porto Alegre: Artmed, 2009.

CIDELL, J. Content clouds as exploratory qualitative data analysis. Area, v. 42, n. 4, p. 514-523, 2010.

CRESWELL, J. W. Projeto de pesquisa: métodos qualitativo, quantitativo e misto. 3. ed. Porto Alegre: Artmed, 2010.

DANTAS, C. C. et al. Teoria fundamentada nos dados - aspectos conceituais e operacionais: metodologia possível de ser aplicada na pesquisa em enfermagem. Revista Latino-Americana de Enfermagem, v. 17, n. 4, p. 573-579, 2009. Disponível em: <http:/ / www.scielo.br/scielo. php?script=sci_arttext\&pid=S0104-11692009000400021\&lng=en\&nrm =iso>. Acesso em: 28 maio 2012.

GIL, A. C. Métodos e técnicas de pesquisa social. 6. ed. São Paulo: Atlas, 2008. 
GLASER, B. Remodeling grounded theory. Forum Qualitative Social Research, v. 5, n. 2, art. 4, 2004. Disponivel em: <http:/ /www.qualitativeresearch.net/index.php/fqs/article/view/607>. Acesso em: 5 ago. 2014.

HOPFER, K. R.; MACIEL-LIMA, S. M. Grounded theory: avaliação crítica do método nos estudos organizacionais. Rev. FAE, Curitiba, v. 11, n. 2, p. $15-24,2008$.

HUGHES, J. A filosofia da pesquisa social. Rio de Janeiro: Zahar Editores, 1983.

NICO, L. S. A. et al. Grounded theory como abordagem metodológica para pesquisas qualitativas em odontologia. Ciência \& Saúde Coletiva, v. 12, n. 3, p. 789-797, 2007.

PEIRCE, C. S. Semiótica e filosofia. São Paulo: Cultrix, 1984.

ROCHA, D.; DEUSDARÁ, B. Análise de conteúdo e análise do discurso: aproximações e afastamentos na (re)construção de uma trajetória. Alea, v. 7, n. 2, p. 305-322, 2005.

SANTOS, B. de S. A crítica da razão indolente: contra o desperdício da experiência. São Paulo: Cortez, 2002.

SANTOS G. E. de O. Cálculo amostral: calculadora on-line. Disponível em: <http:/ / www.calculoamostral.vai.la>. Acesso em: 2 jun. 2012.

SEIDI, M. S. Sinonímia e hiperonímia no sistema e no discurso. In: CÍRCULO DE ESTUDOS LINGUÍSTICOS DO SUL, 8., 2008, Porto Alegre. Anais... Porto Alegre: UFRGS/CELSUL, 2008. 1CD-ROM.

SILVA, M. M. et al. A teoria fundamentada nos dados nos estudos de pósgraduação stricto sensu da enfermagem brasileira. Revista Eletrônica de Enfermagem, v. 13, n. 4, p. 671-679, 2011.

SOUTHWICK, S. B. Biblioteca Digital Brasileira de Teses e Dissertações: modelo e tecnologias. Brasília: IBICT, 2003. 
STEINBOK, D. What is tagCrowd? 2012. Disponivel em: <http://www. tagcrowd.com>. Acesso em: 15 abr. 2012.

STRAUSS, A.; CORBIN, J. Pesquisa qualitativa: técnicas e procedimentos para o desenvolvimento da pesquisa fundamentada. 2. ed. Porto Alegre: Artmed, 2008.

TAROZZI, M. $\mathbf{O}$ que é a Grounded Theory: metodologia de pesquisa e de teoria fundamentada nos dados. Petrópolis: Vozes, 2011.

TRIVIÑOS, A. N. S. Introdução à pesquisa em ciências sociais: a pesquisa qualitativa em educação. 1. ed. 20 reimpr. São Paulo: Atlas, 2011.

VERGARA, S. C.; CALDAS, M. P. Paradigma interpretacionista: a busca da superação do objetivismo funcionalista nos anos 1980 e 1990. RAE, v. 45, n. 4, p. 66-72, 2005. 